Pacific Journal of Mathematic 


\title{
AN INTRINSIC INEQUALITY FOR LEBESGUE AREA
}

\author{
E. Silverman
}

1. Introduction. An inequality for Lebesgue area which corresponds to the fact that the measure of a quadrilateral is not less than the product of the distances between the two pairs of opposite sides may sometimes be useful for the study of this area. This inequality is an extension of a result of Besicovitch [2].

The important results of Cesari [4] and Federer [8] showing the equivalence of Geöcze and Lebesgue area will be used to show that several other 'areas' are equivalent to these two.

This paper depends upon definitions and results of [11] and [12]. In particular we shall use the area defined in [11] which agrees with Lebesgue area for surfaces in Euclidean space.

Let $Q$ be the square $0 \leqq u, v \leqq 1$ having consecutive sides $a, b, c$, and $d$. The set of continuous functions on $Q$ into $m$, the space of bounded sequences [1], will be denoted by $C$, and the family of homeomorphisms of $Q$ into $Q$ by $H$.

Let $x, y \in C$. Then $\tilde{x}$ is defined on $Q \times Q$ to the nonnegative real numbers by

$$
\tilde{x}(p, q)=\|x(p)-x(q)\|
$$

for $(p, q) \in Q \times Q$. If there exists a positive real number $M$ such that

$$
\tilde{x}(p, q) \leqq M\|p-q\|
$$

for all $(p, q) \in Q \times Q$ then $x$ is Lipschitzian. If $\tilde{x}(p, q) \leqq \tilde{y}(p, q)$ for all $(p, q) \in Q \times Q$, then we shall write $\tilde{x} \leqq \tilde{y}$. The Lebesgue area of $x$ is denoted by $L(x)$. If $\tilde{x} \leqq \tilde{y}$ then $L(x) \leqq L(y)$ [Kolmogoroff's principle].

If $i$ and $k$ are distinct positive integers, let $\pi_{\iota k}$ be the plane in $m$ consisting of those points all of whose components, except the $i$ th and $k$ th, are zero. The set of all planes $\pi_{\iota k}$ is $I$. Let $E^{2}$ be the Euclidean plane provided with a cartesian coordinate system, and let $T_{i k}$ be the homeomorphism of $E^{2}$ onto $\pi_{i k}$ defined by

$$
T_{i k}(s, t)=\left\{w^{j}\right\}
$$

where $(s, t) \in E^{2}, w^{i}=s, w^{k}=t$, and $w^{j}=0$ for $i \neq j \neq k$. If $E \subset E^{2}$, and $E$ is Lebesgue measurable, then the measure of $T_{i k}(E),\left|T_{i k}(E)\right|$, is the

Received Miay 2, 1955. 
Lebesgue measure of $E$.

If $x, x_{n} \in C, n=1,2, \cdots$, we write $x_{n} \rightarrow x$ for uniform convergence of $x_{n}$ to $x$. If $f$ and $y$ are two functions with range $f \subset$ domain $g$, then $g f$ is the composition of $f$ and $g$.

In [12] we were interested in geodetic properties of Lebesgue area, and definitions were framed accordingly. Now we are interested in properties which are analogous, equivalent for light mappings, but possibly different in general. The geodetic distance between two points in the parameter square $Q$ is obtained by considering curves in $Q$ joining these two points. In this paper we shall define a pseudo-geodetic distance by considering curves in a "middle-space". Every curve in $Q$ corresponds to a curve in the middle-space, but not conversely. Consequently the pseudo-geodetic distance may be less than the geodetic distance, and the results of [12] will necessarily hold with the modified definition.

If $f$ is continuous on $[0,1]$ into $m$ let $\mu(f)=$ diameter range $f$. If $x \in C$, define $\grave{x}_{\mu}$ on $Q \times Q$ by

$$
\tilde{x}_{\mu}(p, q)=\inf _{\|} \mu(x g) \quad(p, q) \in Q \times Q
$$

for all continuous functions $g$ on $[0,1]$ into $Q$ with $g(0)=p$ and $g(1)=q$. In [12] a function $x_{\mu} \in C$ was constructed such that $\widetilde{\left(x_{\mu}\right)}=\tilde{x}_{\mu}$. Furthermore, if $x_{k} \rightarrow x$ then $x_{k \mu} \rightarrow x_{\mu}$. Finally, $x_{\mu}$ is the monotone factor in a monotone-light factorization of $x$ and has the same Lebesgue area as $x$.

2. An inequality for Lebesgue area. In this section the fundamental inequality of the paper is proved.

Lemma 1. Let $\pi \in ! l$. If $f \in C^{r}$ is Lipschitzian, and range $f \subset \pi$, then $L(f) \geq|f(Q)|$.

Proof. Let $J$ be the Jacobian of $f$, and let $R$ be the set on which $J$ is defined. Then $|Q-R|=0$ and, consequently, $f(Q)|=| f(R) \mid$. Now, by Federer's Theorem [6],

$$
\begin{aligned}
L(f) & =\iint_{Q} J(u, v)\left|d u d v=\iint_{R}\right| J(u, v) d u d v \\
& =\iint_{\pi} N((s, t), f, R) d s d t \geqq|f(R)|=|f(Q)|,
\end{aligned}
$$

where $N((s, t), f, R)$ is the number, possibly infinite, of points $(u, v) \in Q$ such that $f(u, v)=(s, t)$.

If $c$ is an oriented simple closed curve in the plane, and $f$ is continuous on some region containing $c$ into $h^{\prime \prime}$ (the plane of ordered pairs of real numbers with the topology of the Euclidean plane), then for each 
$p \in R^{2}$ let $\mu(p, f, c)$ be the index of $p$ relative to $f$ and $c$ [9, II. 4.34].

Lemma 2 . Let if be continuous from (2 into $R^{2}$. If $\mu\left(p, f, Q^{*}\right) \neq 0$, where $Q^{*}$ is the boundary of $Q$, then $p \in f(Q)$.

This lemma is proved in [9, IV. 1.4-26].

Lemma 3. Let $f=\left(f^{1}, f^{2}\right)$ be continuous from $Q$ into $R^{2}$. Suppose

$$
\begin{aligned}
& p \in a \Rightarrow f^{1}(p)=0, \quad p \in b \Rightarrow f^{2}(p)=0 \text {, } \\
& p \in c \Rightarrow f^{1}(p) \geq r>0, \quad p \in d \Rightarrow f^{\prime \prime}(p) \geq s>0 \text {. }
\end{aligned}
$$

If $y$ and $z$ are real numbers with $0<y<r$ ond $0<z<s$, then $(y, z) \in$ $f(Q)$.

Proof. If we use the notation of Lemma 2 and the result of [9, II. 4.35] we see that $\mu\left(p, f, Q^{*}\right) \neq 0$ if $p=(y, z)$. An application of Lemma 2 completes the proof.

Let $x \in C$ and $x=l M$ be a monotone-light factorization of $x$. If $(p, q)$ $\in Q \times Q$, and $G$ is the set of continuous functions $g$ defined on $[0,1]$ into range $M$ with $g(0)=M(p)$ and $g(1)=M(q)$ then define

$$
\hat{x}_{G i}(p, q)=\inf \{\text { length } l g\} \quad \text { for all } g \in G \text {. }
$$

This definition is independent of the particular factorization of $x$. Finally, if $x=l x_{\mu}$ is the indicated monotone-light factorization of $x$, and $g \in G$ (for $M=x_{\mu}$ ), then

$$
\text { length } l g \leqq \text { length } g \text {. }
$$

From this it follows that

$$
\hat{x}_{G} \leqq\left(\hat{x}_{\mu}\right)_{F_{r}} .
$$

Definition 1. If $x \in C$ define

$$
\alpha(x)=\min _{p \in a, q \in \mathrm{c}} \tilde{x}(p, q) \quad \text { and } \quad A(x)=\inf _{p \in a, q \in a} \hat{x}_{f ;}(p, q) .
$$

Define $\beta(x)$ and $B(x)$ by replacing $a$ by $b$ and $c$ by $d$. Let

$$
\gamma_{i k}=\left\{\min _{0 \leqq v, v \leqq 1}\left|x^{i}(1, v)-x^{i}(0, w)\right|\right\}\left\{\min _{0 \leqq u, v \leqq 1}\left|x^{k}(u, 1)-x^{k}(w, 0)\right|\right\} .
$$

Lemma 4. If $x_{n} \rightarrow x$ in $C$ then $A\left(x_{\mu}\right) \leqq \lim _{n \rightarrow \infty} \inf A\left(x_{n \mu}\right)$.

Proof. We may suppose that for some $M>0, A\left(x_{n \mu}\right)<M$ for all $n$. Hence there exist continuous functions $g_{n}$ on $[0,1]$ into range $x_{n \mu}$ with $g_{n}(0) \in x_{n \mu}(a)$ and $g_{n}(1) \in x_{n \mu}(c)$, such that

$$
\text { length } g_{n}<A\left(x_{n \mu}\right)+1 / n<M+2 \quad \text { for all } n \text {. }
$$


We may now use Hilbert's Theorem to conclude that a subsequence (still denoted by the same subscript) of the $/_{1}$ converges in the sense of Fréchet to a continuous function $g$. Let $p \in$ range $g$. There exist Fréchet equivalent representations $\gamma_{12}$ of $g_{n}$, and a point $t \in[0,1]$, such that $\gamma_{n}(t) \rightarrow p$. Let

$$
P_{n} \in x_{n \mu}^{-1}\left[\gamma_{n}(t)\right] \text {. }
$$

A subsequence of $P_{n}$ (still denoted by the same subscript) will converge to a point $P \in Q$. Then

$$
\begin{aligned}
\left\|p-x_{\mu}(P)\right\| \leqq\left\|p-x_{n \mu}\left(P_{n}\right)\right\| & +\left\|x_{n \mu}\left(P_{n}\right)-x_{\mu}\left(P_{u}\right)\right\| \\
& +\left\|x_{\mu}\left(P_{n}\right)-x_{\mu}(P)\right\| .
\end{aligned}
$$

Thus range $g$ is contained in range $x_{\mu}$. Also $g(0) \in x_{\mu}(a)$ and $g(1) \in x_{\mu}(b)$. Hence

$$
A\left(x_{\mu}\right) \leq \text { length } g \leq \liminf _{n \rightarrow \infty} \text { length } g_{n}=\liminf _{n \rightarrow \infty} A\left(x_{n \mu}\right) .
$$

If $z \in C$ is quasilinear ( $Q$ may be subdivided into a finite number of triangles on each of which $z$ is linear) then there exists a $z_{G} \in C$ such that $z_{t_{i}}$ is Lipschitzian, $\tilde{z}_{f i}=\left(\widehat{z_{\mu}}\right)_{i i}=\hat{z}_{t_{i}}$, and $L(z)=L\left(z_{i i}\right)[12]$.

I.emMa 5. If $z \in C$ is quasilinear then

$$
L(z) \geq A\left(z_{\mu}\right) B\left(z_{\mu}\right)
$$

Proof. It is sufficient to show that

$$
L\left(z_{G}\right) \geq \alpha\left(z_{G}\right) \beta\left(z_{G}\right) .
$$

Let $f$ be the flat transformation from $Q$ to $\pi_{12}$ defined by

$$
f(p)=\left\{f^{j}(p)\right\}
$$

where

$$
f^{1}(p)=\min _{q \in a} \tilde{z}_{G}(p, q), f^{2}(p)=\min _{q \in b} \tilde{z}_{\sigma_{i}}(p, q), f^{j}(p)=0 \quad(j>2) .
$$

If $p, q, p^{\prime} \in Q$, then

$$
\begin{aligned}
& \tilde{z}_{G}(p, q) \leqq \tilde{z}_{G}\left(p, p^{\prime}\right)+\tilde{z}_{G}\left(p^{\prime}, q\right), \\
& \min _{q \in a} \tilde{z}_{G}(p, q) \leqq \tilde{z}_{G}\left(p, p^{\prime}\right)+\min _{q \in a} \tilde{z}_{G}\left(p^{\prime} q\right), \\
& \min _{q \in a} \tilde{z}_{G}(p, q)-\min _{q \in a} \tilde{z}_{G}\left(p^{\prime}, q\right) \leqq \tilde{z}_{G}\left(p, p^{\prime}\right) .
\end{aligned}
$$

The interchange of $p$ and $p^{\prime}$, and of $a$ and $b$, shows that

$$
\left\|f(p)-f\left(p^{\prime}\right)\right\| \leqq \tilde{z}_{G}\left(p, p^{\prime}\right) .
$$

Then by Kolmogoroff's principle and Lemmas 1 and 3, 


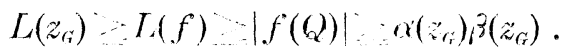

We now make the convention that $0 \cdot \infty=0$.

THEOREM 1. $L(x) \geq A\left(x_{\mu}\right) B\left(x_{\mu}\right) \geq A(x) B(x)$.

Proof. Let $\left\{z_{n}\right\}$ be a sequence of quasilinear functions in $C$ such that $z_{n} \rightarrow x$ and $L\left(z_{n}\right) \rightarrow L(x)$. Then

$$
\begin{aligned}
L(x) & =\lim _{n \rightarrow \infty} L\left(z_{n}\right) \geq \limsup _{n \rightarrow \infty} A\left(z_{n \mu}\right) B\left(z_{n \mu}\right) \\
& \geq\left\{\liminf _{n \rightarrow \infty} A\left(z_{n \mu}\right)\right\}\left\{\liminf _{n \rightarrow \infty} B\left(z_{n \mu}\right)\right\} \\
& \geq A\left(x_{\mu}\right) B\left(x_{\mu}\right) \geq A(x) B(x) .
\end{aligned}
$$

3. Functionals related to Lebesgue area. The alternative definitions of Lebesgue area given in this section may be of some interest to the reader.

For convenience we reserve the letter $F$ to denote a finite subset of $H$ such that ranges of distinct elements of $F$ have no interior points in common.

Definition 2. The functionals $\psi^{*}, \varphi$, and $\Phi$ are defined on $C$ by

$$
\begin{aligned}
& \psi^{*}(x)=\sup _{F^{\prime}} \sum_{h \in F^{\prime}} \sup _{i, k} \gamma_{i k}(x h), \\
& \varphi(x)=\sup _{F^{\prime}} \sum_{h \in F^{\prime}} \alpha(x h)_{i} \beta(x h) \\
& \phi(x)=\sup _{F} \sum_{h \in F^{\prime}} A(x h) B(x h) .
\end{aligned}
$$

THEOREM 2. If $x \in C$ is light and if $\phi(x)<\infty$ then there exists an arc $g$ connecting a and $c$ such that $x g$ is rectifiable.

Proof. If $x$ is light then $x_{\mu}$ is a homeomorphism and $B\left(x_{\mu}\right) \neq 0$. Since $A\left(x_{\mu}\right) B\left(x_{\mu}\right)<\infty$ we can conclude that $A(x) \leqq A\left(x_{\mu}\right)<\infty$, from which the theorem follows.

Let $\Omega$ be any of the three functionals $\psi^{*}, \varphi$, or $\phi$. A familiar argument gives the following.

LEMMA $6 . \quad \Omega(x) \geqq \sum_{h \in F^{F}} \Omega(x h)$.

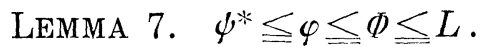

Proof. It is sufficient to recall that $L(x) \geq \sum_{h \in F^{\prime}} L(x h)$.

After we show that $\psi^{*}=L$ for flat transformations we can use the 
results of Cesari and Federer to conclude that the inequalities in the lemma are actually equalities. It will be more convenient for us to use the equality of Peano and Lebesgue rather than that of Geöcze and Lebesgue area. If $x \in C$, then $P(x)$ is the Peano area of $x$. If $L(x)<\infty$ then it is known that $P(x)=L(x)$ [11].

If $N$ is a positive integer let $\pi^{N}$ be the transformation on $m$ into itself determined by

$$
\pi^{N}(t)=\left\{s^{j}\right\}
$$

where

$$
s^{j}= \begin{cases}t^{j} & j \leq N, \\ 0 & j>N .\end{cases}
$$

Lemma 8. $P\left(\pi^{N} x\right)=L\left(\pi^{N} x\right)$.

Proof. Let $T$ map range $\pi^{N}$ into $E^{N}$ by

$$
T\left(\left\{s^{j}\right\}\right)=\{s\} .
$$

Then $T$ and $T^{-1}$ are both Lipschitzian with constants not exceeding $N^{1 / 2}$ and 1 , respectively. Thus if $z \in C$ is quasilinear we can use the definition of area in $m$ [11] to conclude that

$$
(1 / N) \text { area } T \pi^{N} z \leq \text { area } \pi^{N} z \leq \text { area } T \pi^{N} z \text {. }
$$

Thus

$$
(1 / N) L^{\prime}\left(T \pi^{N} x\right) \leqq L\left(\pi^{N} x\right) \leqq L^{\prime}\left(T \pi^{N} x\right)
$$

where $L^{\prime}$ is Lebesgue area in $E^{N}$, and an analogous inequality relates $P$ and $P^{\prime}$, where $P^{\prime}$ is Peano area in $E^{N}$. If $P\left(\pi^{N} x\right)$ is finite then $P^{\prime}\left(T \pi^{N} x\right)$ $=L^{\prime}\left(T \pi^{N} x\right)$ are also, and furthermore, so is $L\left(\pi^{N} x\right)$. From what has already been said we can conclude that $P\left(\pi^{N} x\right)==L\left(\pi^{N} x\right)$.

Let $f$ be continuous from $Q$ into $\pi_{12}$. If $p \in \pi_{12}$ and $\mu(p, f, c) \neq 0$ for a simple closed curve $c$ bounding a Jordan region $J$ contained in $Q$, then there are open oriented rectangles (sides parallel to the coordinate axes) $R^{\prime}$ and $R$ with $p \in R^{\prime} \subset$ closure $R^{\prime} \subset R \subset$ closure $R$ and $\mu(q, f, c) \neq 0$ for all $q \in R$.

Lemma 9. Under the conditions of the preceding paragraph there is a simple closed curve $B$ with $f(B) \subset R$-closure $R^{\prime}$ and such that $\mu(q, f, B)$ $\neq 0$ for all $q \in R^{\prime}$.

Proof. There are only a finite number of components $A_{j}, j=1,2$, $\cdots, n$ of $f^{-1}$ (closure $R^{\prime}$ ) whose intersection with $f^{-1}(p)$ is not empty. The minimum distance between any two of these components is positive 
as is the distance between any of these components and the complement of $f^{-1}(R)$. Hence there exist simple closed curves $c_{j}$ bounding Jordan regions $J_{j}$ with $A_{j} \subset J_{j} \subset f^{-1}(R)$. Some of the curves will not be contained in Jordan regions bounded by any of the other curves. Let $d_{i}$, $i=1,2, \cdots, m$, be this set of curves and denote the regions they bound by $K_{i}$. Suppose that $\mu\left(p, f, d_{i}\right)=0$ for all $i$. By introducing $(m+1)$ arcs joining $c$ to $d_{1}, d_{i}$ to $d_{i+1}$ for $1 \leqq i \leqq m-1$ and $d_{m}$ to $c$, we can decompose $J-\cup K_{i}$ into Jordan regions $L_{1}$ and $L_{2}$ with bounding curves $e_{1}$ and $e_{2}$. Furthermore

$$
0 \neq \mu(p, f, c)-\sum \mu(p, f, d)=\mu\left(p, f, e_{1}\right)+\mu\left(p, f, e_{2}\right) .
$$

Hence either $\mu\left(p, f, e_{1}\right) \neq 0$ or $\mu\left(p, f, e_{2}\right) \neq 0$, which implies that

$$
\begin{array}{r}
0=\cup K_{i} \cap\left(J-\cup K_{i}\right) \supset f^{-1}\left(R^{\prime}\right) \cap\left(J-\cup K_{i}\right) \supset \\
f^{-1}(p) \cap\left(J-\cup K_{i}\right) \neq 0 .
\end{array}
$$

Therefore $\mu\left(p, f, d_{i}\right) \neq 0$ for some $d_{i}$. Let $B$ be this $d_{i}$.

\section{Lemma 10. Let $h \in H$ with $h\left(Q^{*}\right)=B$. Then $\psi^{*}(f h) \geqq\left|R^{\prime}\right|$.}

Proof. Choose $e>0$ and less than (width $R^{\prime}$ )/10. Take $R$ so that the distance between the boundary of $R^{\prime}$ and the boundary of $R$ is less than $e$. Let the sides of $R^{\prime}$ be $X, Y, Z$, and $T$. Since $\mu(p, f, B) \neq 0$ there is a point $q \in B$ such that the distance between $f(q)$ and one side, say $X$, of $R^{\prime}$ is less than $e$. Start from $q$ and traverse $B$ in a positive sense (with respect to the Jordan region $J$ which it bounds). There will be a point $q_{1} \in B$ such that if $r$ is between $q$ and $q_{1}$ (going from $q$ to $q_{1}$ on B) the distance from $f(r)$ to $X$ does not exceed $2 e$, and no point beyond $q_{1}$ has this property. Now start from $q_{1}$ and obtain $q_{2}$ such that for any point $s$ between $q_{1}$ and $q_{2}$ the distance between $f(s)$ and $Y$, a side of $R^{\prime}$ adjoining $X$, does not exceed $2 e$ but that no point beyond $q_{2}$ has this property. (It may be, of course, that points beyond $q_{2}$ have images close to $X$.) In this manner $B$ is divided into a finite number $N$ of closed intervals having only endpoints in common such that the image of a single interval will be within distance $2 e$ of one of the sides of $R^{\prime}$. Since $\mu(p, f, B) \neq 0$, it is necessary that $N>3$.

If $N=4$ take $h \in H$ so that the images of the sides of $Q$ are the four intervals into which $B$ is divided. Then

$$
\gamma_{12}(f h) \geqq(A-4 e)(B-4 \cdot)
$$

where $A$ and $B$ are the lengths of adjoing sides of $R^{\prime}$.

If $N>4$ let $W$ be a strip of width $e$ whose centerline $w$ is parallel to one of the coodinate axes and which divides $R$ into congruent rectang- 
les $S_{1}$ and $S_{2}$. There exists an open rectangle $S^{\prime \prime}$ which contains the closure of $S_{1}$ and for which $\mu(p, f, c) \neq 0$ if $p \in S^{\prime \prime}$. Thus there is a Jordan curve $B^{\prime \prime}$ with $f\left(B^{\prime \prime}\right) \subset S^{\prime \prime}-S_{1}$ and $\mu\left(p, f, B^{\prime \prime}\right) \neq 0$ for $p \in S$. Let $m$ be a component of $B^{\prime \prime}-B$ which contains a point whose image is within a distance $e$ of the center of $R$. If $m$ were all of $B^{\prime \prime}$ we could conclude that $\mu(p, f, B) \neq 0$ for all $p \in f\left(B^{\prime \prime}\right)$, but this is false for any $p \in S_{1}-R^{\prime}$. Thus $m$ connects two points of $B$ whose images lie near opposite sides of $R^{\prime}$ and thus divides $J$ into two Jordan regions $J_{1}$ and $J_{2}$ with bounding curves $B_{1}$ and $B_{2}$. The image of $B_{1}$ is contained in $R-R^{\prime}-W$ as is the image of $B_{2}$.

Two of the intervals into which $B$ was divided, which correspond to opposite sides of $R^{\prime}$, will each have been divided into two smaller intervals by the arc $m$. One of each of these pairs of smaller intervals will belong to $B_{1}$ and the other to $B_{2}$.

The appropriate intervals for $B_{i}$ are all those contained in $B_{i}$ which originally belonged to $B$ plus the arc $m, i=1,2$. Thus

$$
N_{1}+N_{2}=N+4
$$

where $N_{i}$ is the number of intervals into which $B_{i}$ is divided.

For the proof of Lemma 11 we shall wish to remember that $J_{1}$ and $J_{2}$ have no interior points in common.

If $N_{1}=4$ and $N_{2}=4$ then $h_{1}$ and $h_{2}$ can be defined as was $h$ in the case $N=4$. If $W$ were chosen so as to make $A / 2$ and $B$ the width and length of the rectangles into which $R^{\prime}$ was divided then

$$
\gamma_{12}\left(f h_{1}\right)+\gamma_{12}\left(f h_{2}\right)>(A-5 e)(B-4 e) .
$$

If either $N_{1}>4$ or $N_{2}>4$, the preceding procedure can be repeated using a sufficiently narrow dividing strip. Since $N$ is finite we can conclude that $\psi^{*}(f h) \geqq\left|R^{\prime}\right|$.

Lemma 11. If $h \in H, B=h\left(Q^{*}\right), G=\{p \mid \mu(p, f, B) \neq 0\}$, then $\psi^{*}(f h)$ $\geq|G|$.

Proof. Choose $e>0$. Let $R_{i}, i=1,2, \cdots, N$, be a finite set of nonoverlapping open (oriented) rectangles contained in $G$ with $\left|\cup R_{i}\right|>|G|-e$. For each $i$ take another such rectangle $R_{i}^{\prime}$ whose closure is contained in $R_{i}$ and such that $\left|R_{i}^{\prime}\right|>\left|R_{i}\right|-e \mid N$. By Lemmas 9 and 10 there exist $h_{i} \in H$ with $\psi^{*}\left(f h_{i}\right) \geq\left|R_{i}^{\prime}\right|$ and $B_{m} \cap B_{n}=0$ for $m \neq n$ where $B_{i}=h_{i}\left(Q^{*}\right)$. Let $J_{i}$ $=h_{i}(Q)$. If we knew that $J_{m} \cap J_{n}=0$ the lemma would follow since we could take $F$ to be this collection of $h$.

Suppose that $J_{m} \cap J_{n} \neq 0$. Since $B_{m} \cap B_{n}=0$, we may assume that $J_{m} \subset J_{n}$. Fix $w>0$ and use the method of the proof of Lemma 10 to bisect $R_{n}$ into rectangles $S_{1}$ and $S_{2}$ containing rectangles $S_{1}^{\prime}$ and $S_{2}^{\prime}$ respec- 
tively such that

(i) $\left|S_{1}^{\prime}\right|+\left|S_{2}^{\prime}\right|>\left|R_{n}^{\prime}\right|-w$,

(ii) If $K_{1}$ and $K_{2}$ are appropriate Jordan regions for $S_{1}$ and $S_{2}$ then $K_{1} \cup K_{2}=J_{n}$ and $K_{1}$ and $K_{2}$ have no interior points in common.

In a similar manner we can subdivide $S_{1}^{\prime}$ and $S_{2}^{\prime}$. Take $j$ so that $\left|R_{n}\right| / 2^{j}<w$. The area of a rectangle of the collection $\mathscr{S}$ into which $R_{n}$ is divided will be less than $w$. Let $\mathscr{S}^{\prime}$ be a collection of smaller rectangles, one contained in each element of $\mathscr{S}$. Let $\mathscr{K}$ be the collection of associated Jordan regions. We can take $\mathscr{S}^{\prime}$ and $\mathscr{K}$ so that

(i) $\quad \bigcup_{S^{\prime} \in \mathcal{S}^{\prime}} S^{\prime}|>| R_{n}^{\prime} \mid-w$,

(ii) distinct elements of $\mathscr{K}$ have no interior points in common and $\bigcup_{K \in \mathcal{K}} K=J_{n}$.

Let $H_{n}=\{h \in H \mid$ range $h \in \mathscr{\mathscr { C }}\}$. For some $h \in H_{n}, J_{m} \subset$ range $h$. Let $h^{*}$ be one such $h$. Now put $\mathscr{H}=H_{n}-\left\{h^{*}\right\}$. Then

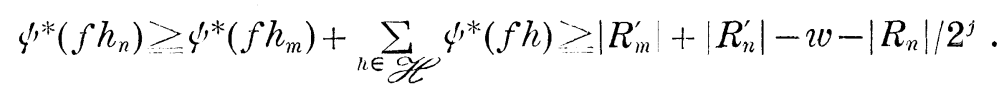

Thus

$$
\psi^{*}\left(f h_{n}\right) \geq\left|R_{m}^{\prime}\right|+\left|R_{n}^{\prime}\right| \text { if } J_{m} \subset J_{n} .
$$

In a similar manner we can conclude that if

$$
h_{m_{j}}(Q) \subset h_{n}(Q) \quad j=1,2 . \cdots, M
$$

then

$$
\psi^{*}\left(f h_{n}\right) \geq \sum_{j=1}^{M}\left|R_{m_{j}}^{\prime}\right|+\left|R_{n}^{\prime}\right| .
$$

Now let $F$ consist of those $h_{m}$ for which range $h_{m}$ is not contained in range $h_{n}$ for $m \neq n$. Then

$$
\psi^{*}(f) \geq \sum_{h \in F} \psi^{*}(f h) \geq \sum\left|R_{i}^{\prime}\right|>|G|-2 e .
$$

LEMMA $12 . \quad \psi^{*}(f)=L(f)$

Proof. By a result of Cesari [5], similar to results of Rado and Reichelderfer [10] and Federer [6],

$$
L(f)=\sup _{F} \sum_{h \in F}\left|G_{h}\right|
$$

where

$$
G_{h}=\left\{p \mid \mu\left(p, f, h\left(Q^{*}\right)\right) \neq 0\right\} .
$$


Thus, by the preceding lemma,

$$
\psi^{*}(f) \leqq L(f)=\sup _{F} \sum_{h \in F}\left|G_{h}\right| \leqq \sup _{F^{\prime}} \sum_{h \in F} \psi^{*}(f h) \leqq \psi^{*}(f) .
$$

THEOREM 3. $P=\psi^{*}=\varphi=\Phi=L$.

Proof. We saw in [11] that $L(x)=\lim _{N \rightarrow \infty} L\left(\pi^{N} x\right)$ and $P(x)=\lim _{N \rightarrow \infty} P\left(\pi^{N} x\right)$. By Lemma 9 we can conclude that $P=L$. Since $\psi^{*}=L=P$ for flat transformations, we have that $P(x) \leqq \psi^{*}(x)$ for all $x \in C$. Thus

$$
L=P \leqq \psi^{*} \leqq \varphi \leqq \Phi \leqq L .
$$

Let $\tilde{x}_{G}(p, q)$ be the geodetic distance between $p$ and $q$, that is, $\tilde{x}_{G}(p, q)=\inf$ length $x g$ for all $g$ continuous on $[0,1]$ into $Q$ with $g(0)=p$ and $g(1)=q$. According to Busemann [3] an area $S$ is intrinsic if $S(x)$ $=S(y)$ whenever $\tilde{x}_{G}=\tilde{y}_{\sigma}$. Let $C_{l}=\{x \in C \mid x$ is light $\}$. If we use the definition of $\Phi$ and the fact that $x_{\mu}$ is a homeomorphism if $x \in C_{l}$ we see that $\Phi$, and therefore $L$, is intrinsic on $C_{l}$.

Choquet suggested an inequality stronger than that the author was able to prove. His inequality would be that of Theorem 1 if geodetic rather than pseudo-geodetic distances were used.

\section{REFERENCES}

1. S. Banach, Theorie des operations lineaires, Warsaw, 1932.

2. A. S. Besicovitch, On two problems of Loewner, J. London Math. Soc., 27 (1952), $141-144$.

3. H. Busemann, Intrinsic areas, Ann. of Math., 48 (1947), 234-267.

4. L. Cesari, Frechct surfaces of finite Lebesgue area in $E_{n}$, Abstract 604, Bull. Amer. Math. Soc., 59 (1953), 534.

5. ____ Sui fondamenti geomelrici dell'integrale classico per l'area delle superficie in forma parametrica, Atti della R. Accademia d'Italia, 13 (1943), 1323-1481.

6. H. Federer, Surface area II, Trans. Amer. Math. Soc., 55 (1914), 438-456.

7. __._. Essential multiplicity and Lebesgue area, Proc. Nat. Acad. Sci. U.S.A. 34 (1948), 611-616.

8. _____ On Lebesgue area, Ann. of Math., 61 (1955), 289-353.

9. T. Rado, Length and area, Colloquium Publications Amer. Math. Soc., 30, New York, 1948.

10. T. Rado and P. V. Reichelderfer, A theory of absolutely continuous transformations in the plane, Trans. Amer. Math. Soc., 49 (1941), 253-307.

11. E. Silverman, Definitions of Lebesgue area for surfaces in metric spaces, Riv. Mat. Univ. Parma, 2 (1951), 47-76.

12. An intrinsic property of Lebesgue area, Riv. Mat. Univ. Parma, 2 (1951), 195-201. 


\section{PACIFIC JOURNAL OF MATHEMATICS}

EDITORS

\author{
H. L. Royden \\ Stanford University \\ Stanford, California \\ E. Hewite \\ University of Washington \\ Seattle 5 , Washington
}

\author{
R. P. Dilworth \\ California Institute of Technology \\ Pasadena 4, California
}

\author{
A. Horn* \\ University of California \\ Los Angeles 24, California
}

\section{ASSOCIATE EDITORS}
E. F. BECKENBACH
M. HALL
M. S. KNEBELMAN
J. J. STOKER
C. E. BURGESS
P. R. HALMOS
I. NIVEN
V. GANAPATHY IYER
T. G. OSTROM
G. SZEKERES
H. BUSEMANN
R. D. JAMES
M. M. SCHIFFER
F. WOLF
H. FEDERER

\section{SPONSORS}

\author{
UNIVERSITY OF BRITISH COLUMBIA \\ CALIFORNIA INSTITUTE OF TECHNOLOGY \\ UNIVERSITY OF CALIFORNIA \\ CALIFORNIA RESEARCH CORPORATION \\ MONTANA STATE UNIVERSITY \\ UNIVERSITY OF NEVADA \\ OREGON STATE COLLEGE \\ UNIVERSITY OF OREGON \\ UNIVERSITY OF SOUTHERN CALIFORNIA
}

\author{
STANFORD UNIVERSITY \\ UNIVERSITY OF UTAH \\ WASHINGTON STATE COLLEGE \\ UNIVERSITY OF WASHINGTON \\ AMERICAN MATHEMATICAL SOCIETY \\ HUGHES AIRCRAFT COMPANY
}

Mathematical papers intended for publication in the Pacific Journal of Mathematics should be typewritten (double spaced), and the author should keep a complete copy. Manuscripts may be sent to any of the editors. Manuscripts intended for the outgoing editors should be sent to their successors. All other communications to the editors should be addressed to the managing editor, Alfred Horn at the University of California, Los Angeles 24, California.

50 reprints of each article are furnished free of charge; additional copies may be obtained at cost in multiples of 50 .

The Pacific Journal of Mathematics is published quarterly, in March, June, September, and December. The price per volume (4 numbers) is $\$ 12.00$; single issues, $\$ 3.50$. Back numbers are available. Special price to individual faculty members of supporting institutions and to individual members of the American Mathematical Society: $\$ 4.00$ per volume; single issues, $\$ 1.25$.

Subscriptions, orders for back numbers, and changes of address should be sent to Pacific Journal of Mathematics, c/o University of California Press, Berkeley 4, California.

Printed at Kokusai Bunken Insatsusha (International Academic Printing Co., Ltd.), No. 10, 1-chome, Fujimi-cho, Chiyoda-ku, Tokyo, Japan.

* During the absence of E. G. Straus.

PUBLISHED BY PACIFIC JOURNAL OF MATHEMATICS, A NON-PROFIT CORPORATION COPYRIGHT 1956 BY PACIFIC JOURNAL OF MATHEMATICS 


\section{Pacific Journal of Mathematics}

\section{Vol. 6, No. $2 \quad$ December, 1956}

Louis Auslander, Remark on the use of forms in variational calculations .......................................... 209

Hubert Spence Butts, Jr. and Henry B. Mann, Corresponding residue systems in algebraic number fields ........................ 211

L. Carlitz and John Herbert Hodges, Distribution of matrices in a finite field............................................

Paul Civin and Bertram Yood, Invariant functionals ............... 231

David James Dickinson, Henry Pollak and G. H. Wannier, On a class of polynomials orthogonal over a denumerable set .................

Bernard Friedman and Luna Mishoe, Eigenfunction expansions associated with a non-self-adjoint differential equation ....................

Luna Mishoe and G. C. Ford, On the uniform convergence of a certain eigenfunction series .............................. 271

John W. Green, Mean values of harmonic functions on homothetic curves...........................................

Charles John August Halberg, Jr. and Angus E. Taylor, On the spectra of linked operators .....................................

Chuan Chih Hsiung, Some integral formulas for closed hypersurfaces in Riemannian space ................................... 291

Norman D. Lane, Differentiable points of arcs in conformal n-space 301

Louis F. McAuley, A relation between perfect separability, completeness, and normality in semi-metric spaces

G. Power and D. L. Scott-Hutton, The slow shearing motion of a liquid past a semi-infinite plane .............................

A. C. Schaeffer, Entire functions

Edward Silverman, An intrinsic inequality for Lebesgue area...

Choy-Tak Taam, Asymptotic relations between systems of differential equations.

Ti Yen, Quotient algebra of a finite $A W^{*}$-algebra ... 